\title{
Erratum
}

\section{Writing systems: Not optimal, but good enough - Erratum}

\author{
Mark S. Seidenberg \\ doi:10.1017/S0140525X12000337, Published online by Cambridge University Press 29 August 2012
}

On page 306 of the commentary by Mark S. Seidenberg on the target article by Ram Frost in the October 2012 issue of Behavioral and Brain Science, there are two text passages that contain grammatical errors introduced during copy editing. The corrected text is as follows:

Reading comprehension is a constant that is maintained via trade-offs between orthographic complexity ("depth," number and complexity of symbols, etc.) and spoken language complexity (particularly morphological). So, in Serbo-Croatian, you, the learner, get the spelling-sound correspondences for free, but then you'll spend years mastering the ferocious inflectional system.

Frost writes that "nothing is arbitrary when it comes to orthographic structure" (sect. 3.2.3, para. 2), but in fact many things are (e.g., the arbitrary association between a visual sign and a pronunciation; for many words in languages such as English and French, which of several possible spellings happens to be used).

We regret the errors.

\section{Reference}

Seidenberg, M. S. (2012) Writing systems: Not optimal, but good enough. Behavioral and Brain Sciences 35(5):305-307. doi:10.1017/S0140525X12000337. 